\title{
El conocimiento de profesionales de la salud sobre la prevención del cáncer cervical. Alternativas de educación médica*
}

\author{
Edna Arillo-Santillán, Lic. en C. de la Educ., (1) Eduardo Lazcano-Ponce, M.C., M. en C., D r. en C., ${ }^{(2)}$ \\ Merce Peris, M.C., M.S.P., ${ }^{(3)}$ Eduardo Salazar-Martínez, M.C., M. en C., ${ }^{(2)}$ \\ Jorge Salmerón-Castro, M.C., M. en C., Dr. en C., ${ }^{(4)}$ Patricia Alonso-De Ruiz, M.C. ${ }^{(5)}$
}

\begin{abstract}
A rillo-Santillán E, Lazcano-Ponce E, Peris M, Salazar-Martínez E, Salmerón-Castro J, Alonso-De Ruiz P. El conocimiento de profesionales de la salud sobre la prevención del cáncer cervical. Alternativas de educación médica. Salud Publica Mex 2000;42:34-42.
\end{abstract}

\section{Resumen}

Objetivo. Evaluar el nivel de conocimientos que diversos tipos de profesionales de la salud tienen sobre la prevención del cáncer cervical. Material y métodos Se realizó un estudio transversal entre 520 profesionales de la salud del estado de Morelos durante 1998, para evaluar y cuantificar el nivel de conocimientos que poseen en relación con el impacto, la etio logía, el tamizaje, el diagnóstico y el tratamiento del cáncer cervicouterino. Se aplicó un cuestionario con escala de 1 a 10, y la muestra incluyó a especialistas en medicina familiar, especialistas de áreas clínicas, enfermeras especialistas y generales, así como trabajadoras sociales. El análisis estadístico incluyó análisis de varianza y estimación de intervalos de confianza al 95\%. Resultados La media del nivel de conocimiento acerca de la prevención del cáncer cervicouterino fue de 4.74 , con un intervalo de confianza (IC) al 95\% de 4.57-4.88, en una escala máxima de 10. La mayor diferencia en el índice de conocimiento acerca de el diagnóstico, la prevención y el tratamiento del cáncer cervical se observó entre los médicos de especialidades clínicas $(X=5.21$, IC $95 \% 4.81-5.60)$, en comparación con las trabajadoras so ciales ( $X=3.07$, IC $95 \% 2.31-3.82$ ). En la mayoría de los casos, la periodicidad con la que una mujer debe

\author{
Arillo-Santillán E, Lazcano-Ponce E, \\ Peris M, Salazar-Martínez E, \\ Salmerón-Castro J, A lonso-De Ruiz P. \\ Knowledge of healthcare professionals on cervical \\ cancer prevention. Alternatives \\ for medical education. \\ Salud Publica Mex 2000;42:34-42.
}

\begin{abstract}
A bstract
Objective. Several studies have shown the importance of health care professionals as predictors of the use of cervical cancer screening (CCS). Material and methods A cross- sectional study of 520 health care professionals in the State of Morelos during 1998, in order to evaluate and quantify their level of knowledge on the impact, etiology, screening, diagnosis and treatment of cervical cancer.A 1 to 10 scale questionnaire was given, and the sample included family medicine specialists, general practitioners, specialist and general nurses, and social workers. Statistical analysis included analysis of variance and $95 \%$ confidence intervals. Results.A knowledge mean of $4.74(95 \% \mathrm{Cl}$ 4.57-4.88) was observed for a scale of 10; specialist physicians scored higher (mean $5.21,95 \% \mathrm{Cl} 4.81-5.60$ ) than so cial workers (mean $3.07,95 \% \mathrm{Cl}$ 2.31-3.82). Periodicity of the Pap test in most cases was less than 1 year and there was poor consensus about the age period during which the Pap test should be obtained. The knowledge level was lower when trying to identify etiologic aspects and treatment perspectives. Conclusions. The results of this study show that, besides the improvement of undergraduate academic programs, it is necessary to improve educational interventions for health
\end{abstract}

* Este trabajo fue desarrollado gracias a una beca de la Unión Internacional Contra el Cáncer (ICRETT, N 0. 953), así como a la colaboración del Instituto C atalán de 0 ncología, el Instituto Mexicano del Seguro Social, la Secretaría de Salud del Estado de Morelos y el Instituto N acional de Salud Pública.

(1) Secretaría A cadémica, Instituto N acional de Salud Pública (IN SP), México.

(2) Centro de Investigación en Salud Poblacional, IN SP, México.

(3) Instituto Catalán de O ncología, Barcelona, España.

(4) Unidad de Investigación Epidemiológica, Instituto Mexicano del Seguro Social, Centro Médico N acional Siglo XXI, México.

(5) Unidad de Patología, Universidad N acional Autónoma de México. Hospital General de México, México.

Fecha de recibido: 9 de julio de 1999 - Fecha de aprobado: 29 de noviembre de 1999

Solicitud de sobretiros: Eduardo C. Lazcano Ponce. Centro de Investigación en Salud Poblacional, Instituto N acional de Salud Pública. Universidad 655, colonia Santa María A huacatitlán, 62508 Cuernavaca, Morelos, México.

Correo electrónico: elazcano@ insp3.insp.mx 
hacerse la prueba de Papanicolaou se postula menor a un año, y existe un pobre consenso en relación con el periodo de edad en que debe obtenerse dicha prueba. El nivel de conocimientos es menor conforme se tratan de identificar aspectos etiológicos y perspectivas de tratamiento. Conclusiones. La educación médica continua es necesaria para el desarrollo óptimo de un programa de detección oportuna de cáncer cervical. Los resultados del estudio en el estado de Morelos revelan que, además de mejorar los programas académicos de formación de pregrado, es necesario incrementar las intervenciones educativas entre los profesionales de la salud. Para ello, se contemplan diversas estrategias, entre las que destacan la actualización, el reciclaje, la capacitación, la educación en salud y la educación médica continua. De esta forma se podrá promover la competencia profesional para incrementar la calidad de la atención médica.

Palabras clave: personal de salud/conocimiento; neoplasmas del cuello uterino; tamizaje masivo; educación continua; México care professionals through the updating, recycling, training, health education, and continuing medical education, among others, to promote professional competence and thus improve the quality of medical care.

Key words: health personnel/awareness; cervix neoplasms; mass screening; education, continuing; Mexico os requerimientos para que un programa organizado de detección oportuna de cáncer cervical (PDOC) sea eficiente han sido descritos ampliamente por diversos autores. ${ }^{1,2}$ Todos ellos señalan la necesidad de alcanzar una elevada cobertura entre las mujeres en riesgo, así como la de introducir un control de calidad en cada uno de los elementos del PDOC. ${ }^{3,4}$ Las principales iniciativas desarrolladas al respecto han sido: a) la definición de estrategias de búsqueda y captación de mujeres de alto riesgo; b) la prestación de un servicio accesible y aceptable para la toma de especímenes; c) la obtención de una elevada calidad en la toma de muestras, el diagnóstico y el tratamiento; d) el manejo apropiado de mujeres con anormalidades detectadas y, finalmente, e) la realización de un monitoreo y una evaluación continua del PDOC. En este contexto, se han descrito diversas estrategias para promover la participación de las mujeres en riesgo dentro de programas de tamizaje en cáncer cervicouterino $(\mathrm{CaCu})$.

Una de las principales alternativas denominadas oportunistas, es la promoción del Papanicolaou en los servicios de atención médica. Un elevado número de estudios han mostrado la importancia de los profesionales de la salud como predictores de la utilización del tamizaje; diversos autores ${ }^{5,6}$ han señalado que el hecho de que las mujeres en riesgo acudan a un servicio de atención médica, amplía la oportunidad de brindarles educación acerca de la prevención del $\mathrm{CaCu}$, lo que a su vez influye de manera importante en su decisión de participar en el PDOC.
Trabajos previos han señalado la importancia de promover la educación continua entre los médicos, para que aumenten sus conocimientos y habilidades respecto al tamizaje de cáncer cervical y se conviertan en habilitadores del mismo. ${ }^{7}$ Se esperaría que los profesionales de la salud que participan en los servicios de atención médica, tuvieran un elevado conocimiento sobre las premisas básicas de la prevención del cáncer cervical. El objetivo de esta investigación fue evaluar y cuantificar el grado de conocimientos en una muestra de profesionales de la salud del estado de Morelos, acerca del cáncer cervical, de su etiología, de las medidas de prevención secundaria que deben aplicarse, así como de las alternativas diagnósticas y de las pautas de tratamiento, quienes potencialmente promueven la participación en el PDOC. Como alternativa a los resultados obtenidos, se describen las principales modalidades de educación médica continua que pueden ser implementadas.

\section{Material y métodos}

Durante 1998 se realizó un estudio transversal entre 520 profesionales de la salud que participan en instituciones del primero y segundo nivel de atención en el estado de Morelos.

Mediante un muestreo aleatorio simple irrestricto y considerando una prevalencia de conocimiento eficiente sobre cáncer cervical de $70 \%$, se estimó un tamaño de muestra inicial de 420 profesionales de la salud. La muestra de estudio quedó conformada por: a) médi- 
cos que participan en la atención primaria como especialistas de medicina familiar en el Instituto Mexicano del Seguro Social (IMSS) de la Delegación Morelos; b) especialistas y residentes de áreas clínicas del Hospital General Dr. José Parres, del Hospital del Niño Morelense, de la Secretaría de Salud en el estado de Morelos, así como de la Facultad de Medicina de la Universidad Autónoma del Estado de Morelos (UAEM); c) enfermeras especialistas y generales de la Escuela de Enfermería de la UAEM, adscritas a la Delegación Morelos del IMSS, y d) trabajadoras sociales de las unidades de atención médica referidas.

Se entrevistó a 141 especialistas en medicina familiar, 82 especialistas de áreas clínicas, 78 médicos residentes de especialidad, 135 enfermeras generales, 71 enfermeras especialistas y 13 trabajadoras sociales. La tasa de respuesta obtenida fue de $85 \%$, motivo por el cual se incrementó la población calculada inicialmente.

Dos enfermeras generales con estandarización previa aplicaron, de manera confidencial y con el consentimiento informado de los sujetos, un cuestionario de preguntas abiertas. Las entrevistadoras marcaban las opciones que correspondían a las respuestas espontáneas de los sujetos ante las preguntas específicas hechas.

Este cuestionario había sido previamente adaptado -de acuerdo con las normas actuales de detección oportuna de $\mathrm{CaCu}$ en el ámbito nacional- estandarizado y validado en una muestra de profesionales de la salud del Hospital General de México.* Asimismo, se le había cotejado con evaluaciones previas desarrolladas en países con programas organizados de DOC, ${ }^{8}$ donde se establecen premisas básicas del conocimiento sobre el impacto del $\mathrm{CaCu}$, su etiología, su detección temprana y su tratamiento conservador, al igual que sobre las políticas eficientes de tamizaje. ${ }^{9}$ incluían:

El cuestionario fue dividido en dos secciones que

1. Características sociodemográficas, es decir: a) antecedentes académicos, edad y sexo, y b) tipo de especialidad clínica.

2. Reactivos para medir el conocimiento sobre las premisas básicas de detección oportuna de cán-

\footnotetext{
* Arillo-Santillán E , Nigenda G, Sánchez-Prado V M, Alonso-De Ruiz P, Nájera-Aguilar P, Lazcano-Ponce EC. Awareness about cervical cancer prevention in physicians from Mexico. Implications for cancer screening. Enviado a J Cancer Educ.
}

cer, como los siguientes: a) ¿Incluyó el cáncer cervical en la descripción de los cinco principales problemas de salud pública en México?; b) ¿Incluyó el cáncer cervical en las cinco causas de muerte potencialmente prevenibles en mujeres mexicanas?; c) Si le pidieran priorizar, ¿cuál sería el principal agente etiológico del $\mathrm{CaCu}$ ? (se consideró como respuesta correcta "virus del papiloma humano"); d) ¿Cuál es la prueba de tamizaje para la detección oportuna de cáncer cervical? (se consideró como respuesta correcta "citología cervical"); e) De acuerdo con estándares internacionales, ¿cuál es la periodicidad de la prueba de Papanicolaou? (entre 1 y 3 años); f) Con estándares internacionales, ¿cuáles son los grupos de edad ideales para iniciar y terminar el tamizaje en cáncer cervical? (entre 25 y 65 años); g) Conocimiento de, al menos, una nomenclatura para el diagnóstico de citología ginecológica (displasias -Organización Panamericana de la Salud-, neoplasia intraepitelial cervical -NIC-, Papanicolaou y/o Sistema Bethesda); h) Conocimiento de, al menos, una condición necesaria en las mujeres para poder obtener un especimen de Papanicolaou; i) De acuerdo con el conocimiento de la historia natural del cáncer cervical, ¿cuál es el tratamiento conservador utilizado actualmente tras el diagnóstico de lesiones precursoras de cáncer cervical? (se consideraron como respuestas correctas cualesquiera de las siguientes: "ablasión, conización; crioterapia, laserterapia o destrucción de la zona de transformación con asa diatérmica"); j) ¿Cuál es el tratamiento para la infección del virus del papiloma humano $(\mathrm{VPH})$ en ausencia de lesión cervical? (se consideró como respuesta correcta "no hay evidencia de un tratamiento efectivo actual").

3. Asimismo, se incluyó una pregunta en la que se pidió priorizar un factor atribuible a la ineficiencia del PDOC en países como México.

Se realizó un análisis univariado y con ANOVA, utilizando el programa estadístico Stata. ${ }^{10}$ El procedimiento estadístico se llevó a cabo mediante una prueba paramétrica de análisis de varianza y con la estimación de intervalos de confianza al 95\%.

Se construyó un índice de conocimiento sobre prevención de cáncer cervical, considerando las respuestas en una escala de 1 a 10 y asignando un punto por cada una de las preguntas respondidas, al menos dentro de los parámetros de estimación correcta previamente establecidos; se calcularon los intervalos de confianza al $95 \%$. 


\section{Resultados}

Entre los 520 profesionales de la salud estudiados, la media de conocimiento total fue de 4.74 puntos (IC95\% 4.57-4.88) en una escala máxima de 10; el grado de conocimiento entre los médicos de especialidades clínicas fue mayor ( $\mathrm{X}=5.21$, IC95\% 4.81-5.60), en comparación con las trabajadoras sociales ( $\bar{X}=3.07$, IC95\% 2.31-3.82) (cuadro I).

Como se puede observar en la figura 1, la muestra de profesionales de la salud del estado de Morelos considera, un porcentaje menor a $55 \%$, que el cáncer cervical es un problema de salud pública en México ( $30 \%$ de las trabajadoras sociales, en comparación con
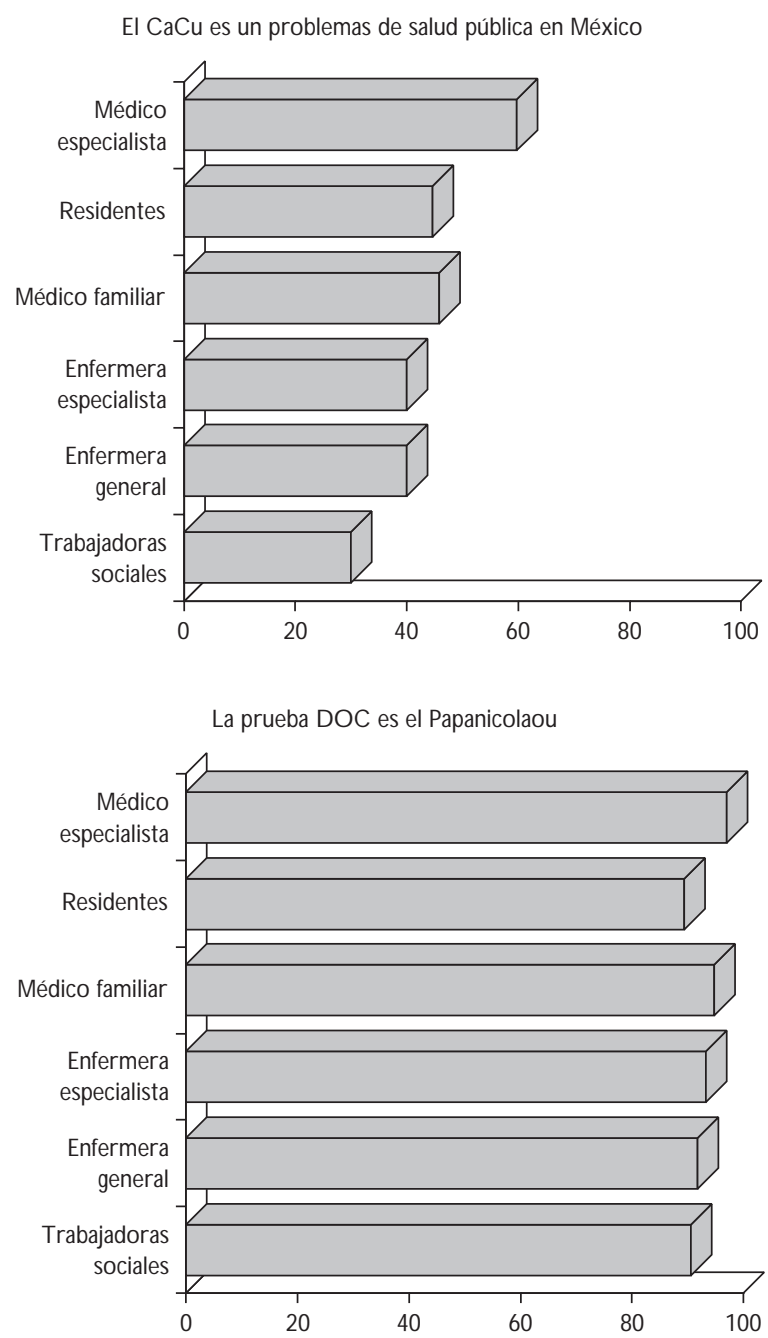

$60 \%$ de médicos especialistas). Asimismo, la mayoría identifica la prueba de tamizaje como medio para la prevención secundaria de cáncer cervical. Los médicos especialistas y los familiares identifican claramente al menos una nomenclatura diagnóstica, en comparación con las trabajadoras sociales $(0 \%)$, enfermeras generales $(40 \%)$, enfermeras especialistas $(58 \%)$ y médicos residentes $(56 \%)$.

Los datos muestran que existe un mayor conocimiento sobre las condiciones necesarias para la obtención del Papanicolaou entre enfermeras especialistas (76\%) y generales $(74.8 \%)$, en comparación con los médicos familiares (32.6\%). La periodicidad con la que las mujeres deben hacerse la prueba de Papanicolaou,
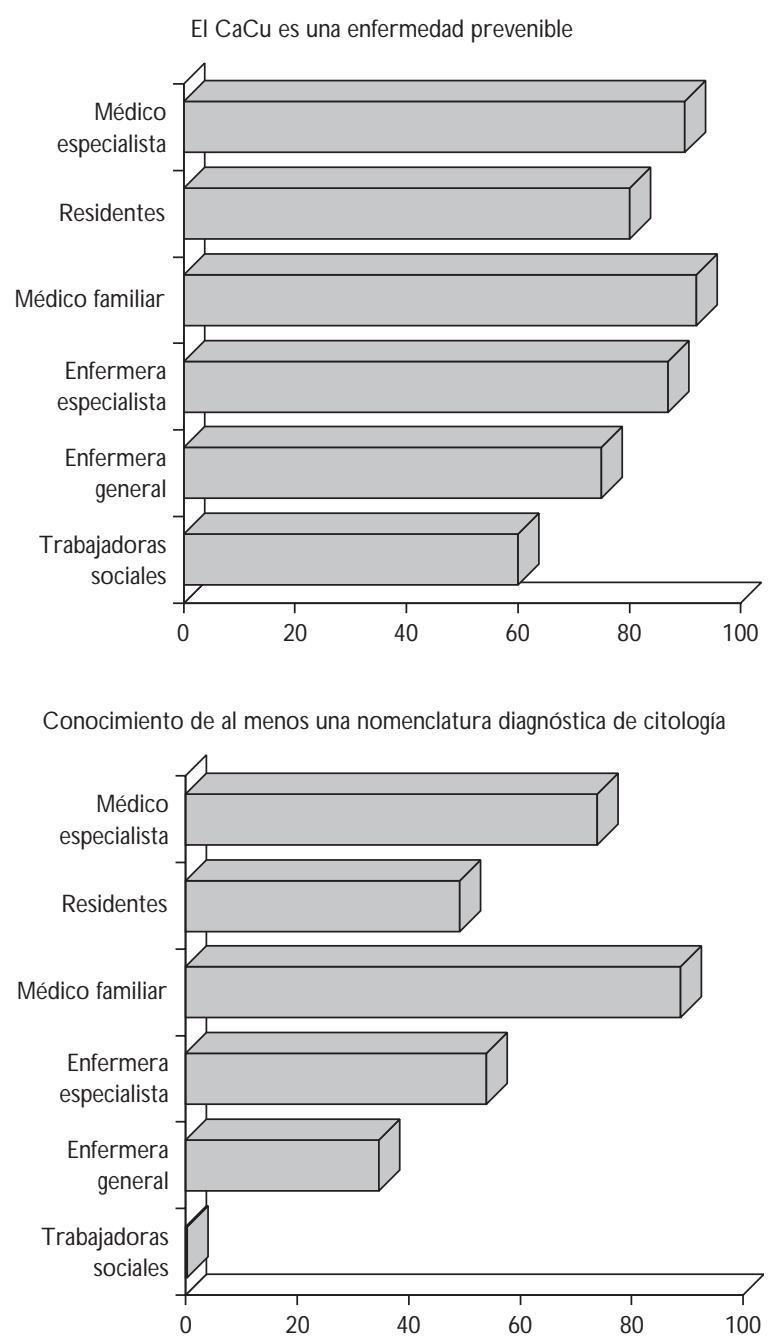

Figura 1. Porcentaje de respuestas afirmativas aCerCa del impacto y la preVención de CánCer Cervical entre PRofesionales de la SALud. Morelos, MéXico, 1998 


\section{Cuadro I}

INDICE DE CONOCIMIENTO* DE LA MAGNITUD, EL DIAGNÓSTICO, LA PREVENCIÓN Y EL TRATAMIENTO del CÁncer Cervical en profesionales de la salud. Morelos, México, 1998

\begin{tabular}{lccccc} 
Ocupación & Número & Media & IC95\% & Valor mínimo & Valor máximo \\
Trabajadora social & 13 & 3.07 & $2.31-3.82$ & 1 & 5 \\
\hline Enfermera general & 135 & 4.32 & $4.03-4.60$ & 0 & 8 \\
\hline Enfermera especialista & 71 & 4.94 & $4.57-5.30$ & 2 & 8 \\
\hline Especialista en medicina familiar & 141 & 5.11 & $4.86-5.35$ & 2 & 9 \\
\hline Residentes de especialidad & 78 & 4.42 & $4.06-4.77$ & 1 & 9 \\
\hline Médicos especialistas & 82 & 5.21 & $4.81-5.60$ & 2 & 9 \\
\hline Total & 520 & 4.74 & $4.57-4.88$ & 0 & 9
\end{tabular}

*Indice construido de 1 a 10 como máximo

Modelo AN O VA, $f=8.34, p<0.001$

Cuadro II

Conocimiento de los profesionales de la salud sobre la prevención del cáncer cervical. Morelos, MÉxico, 1998

\begin{tabular}{|c|c|c|c|}
\hline Ocupación & $\begin{array}{c}\text { Conoce al menos una condición previa } \\
\text { a la toma del Papanicolaou } \\
\%\end{array}$ & $\begin{array}{c}\text { Describe una periodicidad de aplicación } \\
\text { del Papanicolaou de entre } 1 \text { a } 3 \text { años } \\
\text { \% }\end{array}$ & $\begin{array}{c}\text { Periodo de edad en que debe tomarse } \\
\text { un Papanicolaou ( } 25 \text { a } 65 \text { Años) } \\
\%\end{array}$ \\
\hline Trabajadora social & 53.8 & 7.69 & 15.3 \\
\hline Enfermera general & 74.8 & 2.2 & 25.2 \\
\hline Enfermera especialista & 76.0 & 0 & 23.9 \\
\hline Especialista en medicina familiar & 32.6 & 0.7 & 22.7 \\
\hline Residentes de especialidad & 50.5 & 0 & 14.1 \\
\hline Médicos especialistas & 62.2 & 0 & 20.7 \\
\hline
\end{tabular}

en la mayoría de los casos se postula menor a un año, y existe un pobre consenso en torno al periodo de edad en el que se debe obtener dicha prueba (cuadro II).

El nivel de conocimientos es menor conforme se intenta identificar aspectos etiológicos y perspectivas de tratamiento. En la figura 2 se observa que $40 \%$ de los especialistas en medicina familiar no saben de la asociación entre el VPH y el $\mathrm{CaCu}$, en comparación con las trabajadoras sociales, quienes la desconocen hasta en $94 \%$. Asimismo, existe un pobre conocimiento sobre el tratamiento conservador ante el diagnóstico de lesiones precursoras de cáncer invasor y ante la presencia de VPH sin el desarrollo de lesiones cervicales.

Finalmente, llama la atención el hecho de que un alto procentaje de los profesionales de la salud entrevistados haya atribuido a las usuarias la inefectividad del programa de detección oportuna de cáncer (entre 48.94 y $79.49 \%$ ), como se muestra en el cuadro III.

\section{Discusión}

Los determinantes de la participación de las usuarias en el PDOC son complejos, pero uno de los más importantes es la presencia de un profesional de la salud que recomienda o practica el procedimiento. ${ }^{11}$ Los resultados del estudio en el estado de Morelos revelan que, además de mejorar la concepción pedagógica de los programas académicos de formación de pregrado, ${ }^{12}$ es necesario incrementar las intervenciones educativas entre los profesionales de la salud. Para ello, se consideran diversas estrategias, entre las que destacan: la actualización, el reciclaje, la capacitación, la educación en salud y la educación médica continua. De esta forma, se podrá promover la competencia profesional para incrementar la calidad de la atención médica.

Respecto al futuro de la educación médica en el ámbito mundial, se han planteado algunas recomendaciones; una tendencia es la de adaptar la educación 


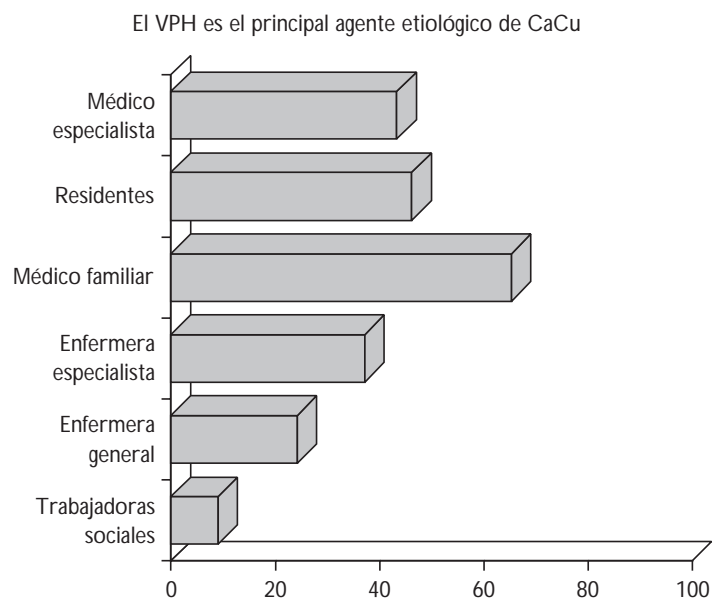

El tratamiento conservador de lesiones precursoras de $\mathrm{CaC}$ u es ablasión, conización, crioterapia, laserterapia o destrucción de la zona de

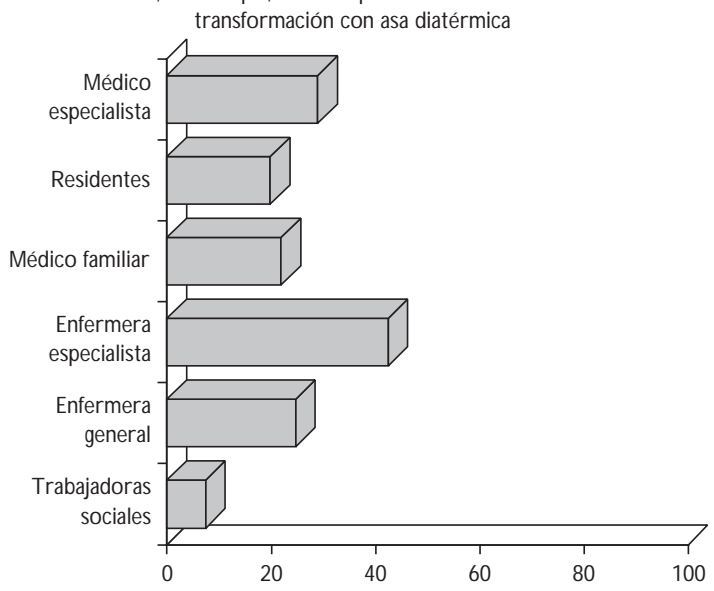

No existe tratamiento para el diagnóstico de infección por VPH

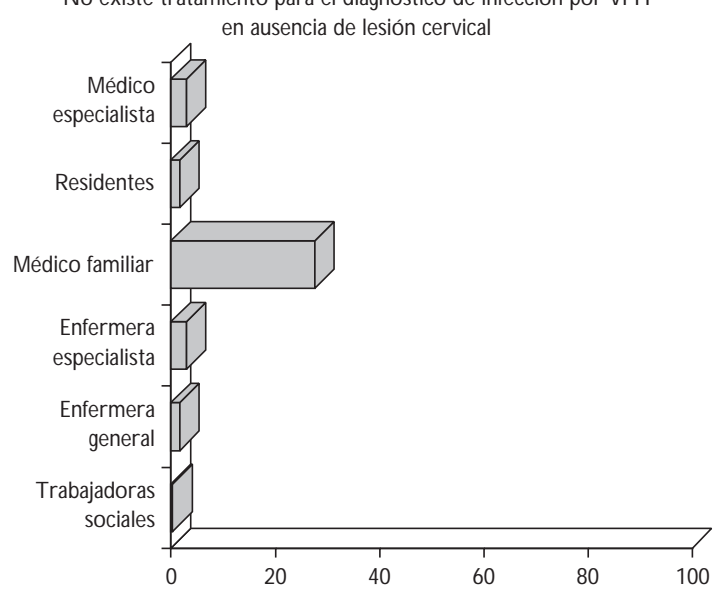

Figura 1. Porcentaje de respuestas afirmativas en RELACIÓN CON LA ETIOLOGÍa Y EL TRATAMIENTO CONSERVador para neoplasia Cervical. Profesionales de la Salud del estado de Morelos, México, 1998

salud pública de méxico / vol.42, no.1, enero-febrero de 2000 continua y el entrenamiento de los profesionales de la salud a las necesidades nacionales y regionales de educación médica en todos sus niveles. ${ }^{13}$ Además, se ha señalado la importancia de que los programas educativos concuerden con la realidad epidemiológica del país, así como con las políticas y los programas nacionales de salud.

La intervención educativa entre los profesionales de la salud es necesaria para promover un incremento en la cobertura de las mujeres en riesgo dentro del PDOC, en conjunción con una gran variedad de propuestas alternativas también necesarias para disminuir las barreras de utilización de los servicios de atención médica. En el futuro será necesario realizar más estudios que conduzcan a revisar la formación académica de diversos profesionales de la salud, en términos no sólo de la calidad con la que son formados, sino también de los contenidos y habilidades descritos en sus planes y programas de estudio, que en teoría deben priorizar los principales problemas de salud pública, tanto en el ámbito regional como en el nacional.

En nuestro trabajo se hace evidente la gran heterogeneidad que existe en el conocimiento que los profesionales de la salud tienen sobre la prevención del cáncer cervical. Por ejemplo, el consenso en cuanto a la periodicidad con la que debe ofertarse la prueba de Papanicolaou es pobre, y existen desventajas inherentes a este hecho; una de ellas es la sobreoferta del tamizaje, producida por la enorme confusión existente respecto al intervalo óptimo que debe haber entre cada prueba, lo que a su vez provoca que varíen los mensajes emitidos dentro de los programas de educación en salud dirigidos a la población en riesgo. Es preciso señalar que evidencias epidemiológicas múltiples han señalado que el tamizaje ofrecido cada tres años reduce en $91 \%$ el riesgo de cáncer invasor, en comparación con el $93 \%$ que arroja la aplicación de un tamizaje anual, a un costo mucho más elevado. ${ }^{14}$

La inclusión de profesionales de la salud con especialidad clínica en el estudio (médicos y enfermeras especialistas, así como residentes de especialidad) se justifica, porque existen evidencias de la importancia de su participación en el tamizaje dentro del ámbito hospitalario. Estudios hechos entre mujeres con cáncer cervical invasor han mostrado que en los años previos al diagnóstico éstas pierden varias oportunidades de tamizaje. MacGregor ${ }^{15}$ notificó en Escocia que 75\% de las mujeres con cáncer cervical invasor, aun cuando habían tenido admisiones hospitalarias previas por otras condiciones, no habían sido tamizadas. Similares hallazgos se obtuvieron entre mujeres con $\mathrm{CaCu}$ de diversas ciudades de Estados Unidos de América. ${ }^{16,17}$ En el caso de México, se ha notificado que más de 50\% de las mujeres que acuden al PDOC refieren no haber 


\section{Cuadro III \\ Factores que influyen en el escaso impacto del programa meXicano de detección oportuna de cáncer, Según profesionales de la SAlud. Morelos, México, 1998}

\begin{tabular}{lccccc} 
Ocupación & Usuario & Servicios & Proveedores & Otro & No sabe \\
Trabajadora social & 69.23 & 0 & 23.08 & 0 & 7.69 \\
\hline Enfermera general & 62.96 & 8.9 & 20.74 & 2.22 & 5.19 \\
\hline Enfermera especialista & 60.56 & 8.45 & 23.94 & 1.41 & 5.63 \\
\hline Especialista en medicina familiar & 48.94 & 11.35 & 14.18 & 6.38 & 19.15 \\
\hline Residentes de especialidad & 79.49 & 6.41 & 7.69 & 3.56 & 3.66 \\
\hline Médicos especialistas & 67.07 & 9.76 & 13.41 & & 6. \\
\hline
\end{tabular}

recibido información de los profesionales de la salud sobre lo que es la prueba de Papanicolaou, o bien, sobre los procedimientos que se siguen para la toma de la muestra y el proceso subsecuente de atención. ${ }^{18}$

Para incrementar la práctica del tamizaje entre los profesionales de la salud se han propuesto varias acciones, y una de ellas es el otorgamiento de incentivos financieros,$^{19}$ lo cual es difícil de aplicar en el contexto latinoamericano. En países como México es necesario considerar diversas estrategias educativas relacionadas con el tamizaje; habría que tomar en cuenta la capacidad de comunicación que tienen los profesionales de la salud para involucrar en los programas de prevención de $\mathrm{CaCu}$ a las mujeres en riesgo, así como sus habilidades para obtener el especimen de Papanicolaou.

En Victoria, Australia, ${ }^{20}$ una evaluación de tales habilidades notifica que cerca de $31 \%$ de los profesionales de la salud no siempre obtienen una muestra cada vez que revisan el cérvix; asimismo, se ha documentado que la mayoría de los especímenes mal tomados se relaciona con la intervención de médicos recién graduados o que han acumulado más de 25 años de experiencia, pero sin adiestramiento continuo. Lewis y colaboradores ${ }^{20}$ señalan, finalmente, que los profesionales de la salud de Victoria tienen escaso conocimiento y pocas habilidades para aplicar el tamizaje de cáncer cervical, tal como lo sugieren los resultados de este trabajo.

Otro aspecto de la educación dirigida a los profesionales de la salud es la retroalimentación respecto a su actuación en programas de detección de cáncer, como lo han descrito estudios hechos en Holanda, ${ }^{21}$ Australia ${ }^{22}$ y Francia, ${ }^{23}$ donde la formación continua de los médicos en servicio debe enfocarse en la sensibilización del profesional hacia la importancia de aplicar el tamizaje en la búsqueda activa de casos. De acuerdo con este punto de vista, el médico de atención primaria debe buscar enfermedades o problemas de salud no relacionados con los signos o síntomas que llevan al paciente a consulta, mediante el interrogatorio, la exploración física y las pruebas complementarias pertinentes; así, una vez que confirma el diagnóstico, el médico puede tomar medidas oportunas.

Los resultados obtenidos en este estudio evidencian que los profesionales de la salud del estado de Morelos requieren de conocer los avances que ha tenido la medicina preventiva en la detección oportuna de cáncer cervical, lo que puede mejorar la eficiencia del PDOC. Para ello es posible utilizar diversas propuestas educativas como la actualización, las intervenciones para introducir al profesional en las actividades que su puesto le exige desarrollar (reciclaje), la capacitación, la educación en salud y la educación médica continua.

La actualización ${ }^{24}$ se refiere a procesos de perfeccionamiento que permitan a aquellos profesionales que participan en el PDOC adquirir conocimientos de vanguardia y desarrollar destrezas y habilidades específicas. La intervención para introducir al profesional en el puesto que asume* se ha definido como la reproducción del conocimiento entre el personal que no tiene experiencia previa en un área específica, pero cuya actividad laboral le demanda tener conocimientos al respecto. Por su parte, la capacitación se refiere a poner en operación inmediata conocimientos dirigidos a mejorar el desarrollo del trabajo. Otro término utilizado para la formación de profesionales de la salud es la educación en salud ${ }^{25}$ que se define como el conjunto de acciones educativas, formales e informales, encaminadas a la formación de recursos humanos, indispensables para atender las necesidades que, en relación con el proceso salud-enfermedad, se generan en una población.

\footnotetext{
* Santos-Burgoa C. Presentación. En: Escuela de Salud Pública de México-Instituto Nacional de Salud Pública. Programa de Educación Continua 1993. México, D.F.: ESPM-INSP, 1992:10.
} 
Finalmente, la educación médica continua ${ }^{26}$ es la que conjunta a estas actividades educativas, organizándolas en cierta continuidad de tiempo; está dirigida al personal de salud que ya se encuentra en ejercicio y cuyo objetivo principal es el de completar su formación inicial.

En todos los casos es fundamental definir las competencias laborales donde intervienen el conocimiento, las actitudes y las habilidades de realización* que todo profesional debe tener respecto a la prevención y el tratamiento del $\mathrm{CaCu}$; ello permitiría obtener un alto grado de flexibilidad y réplica en el proceso educativo, según las necesidades de los participantes y su campo de acción. Por sí misma, la educación médica continua se justifica: en primer lugar, en el campo de la medicina, alrededor de $50 \%$ de los conocimientos tienen una vigencia promedio de 5 a 10 años, lo que hace necesaria la actualización para evitar la obsolescencia del conocimiento. En ese sentido, las políticas nacionales enfocadas a la acreditación de la competencia, obligan a una constante actualización. Asimismo, la curva del olvido es otro factor a considerar, aunado al hecho de que actualmente la oferta de conocimiento es cada vez mayor y al de que la incorporación temprana de este conocimiento a la práctica médica se presenta con más rapidez. ${ }^{27}$

Es preciso señalar también que el desarrollo tecnológico en el área de las telecomunicaciones y la informática -evidenciado por el uso de equipos multimedia y de herramientas de internet como el correo electrónico- hace que el conocimiento esté al alcance no sólo de los profesionales, sino también de la población; así, una sociedad mejor informada exige mayor calidad en los servicios que recibe. ${ }^{27}$

Una de las limitantes para la actualización del profesional en servicio es la disponibilidad de tiempo libre, aunque existen experiencias basadas en la educación a distancia y en materiales de autoaprendizaje, de tal forma que el profesional puede actualizarse a su propio ritmo, de acuerdo con su disponibilidad de tiempo y sin abandonar sus labores rutinarias. ${ }^{28,29}$

Respecto a los métodos y técnicas didácticas, en la enseñanza clínica se han privilegiado las demostraciones y conferencias. Varios investigadores, aplicando

\footnotetext{
* El conocimiento es el resultado de procesos perceptivos y conceptuales, como ayudar, seleccionar, ensayar simbólicamente, descifrar, codificar, reflexionar y evaluar respuestas; las actitudes son los productos de las respuestas emocionales hacia acontecimientos y objetos específicos, y las habilidades de realización se refieren a los resultados de procesos psicomotores que permiten reaccionar de modo manifiesto y crear un producto tangible que pueda ser observado y evaluado por otra persona.
}

la técnica del incidente crítico y el análisis factorial de escalas de estimación, han estudiado lo que los participantes consideran eficaz en la enseñanza; los resultados coinciden en que la participación activa de los estudiantes en actividades centradas en la resolución de problemas, así como las oportunidades de practicar las habilidades adquiridas, figuran entre los componentes que facilitan el aprendizaje. ${ }^{30}$ De hecho, estudios evidencian resultados positivos, además de las ventajas de las propuestas de aprendizaje basado en la resolución de problemas en las escuelas de medicina, aunque también señalan sus limitaciones. . $^{31,32}$

Dentro de las líneas de acción que establece el PDOC nacional, ${ }^{33}$ se encuentran el diseño y la elaboración de materiales dirigidos al personal de salud, con el fin de proporcionarles información y, además, sensibilizarlos sobre la importancia del programa.

Finalmente, si se quiere dar respuesta a las necesidades de formación y actualización de los profesionales de la salud, es necesario: a) diversificar las opciones y estrategias educativas de acuerdo con su perfil profesional; b) privilegiar el desarrollo no sólo de conocimientos, destrezas y habilidades de realización, sino también de la creatividad en la elección de nuevas respuestas a sus prácticas, y c) sensibilizarlos ante un problema de salud pública potencialmente prevenible, como lo es el cáncer cervical.

\section{Agradecimientos}

Agradecemos el apoyo de María de Lourdes Alvarez Díaz y de Lucila Hernández Reyes, por su excelente participación en el desarrollo de la fase de campo y por hacer posible la realización del presente trabajo, que forma parte de su entrenamiento continuo en la Escuela de Enfermería de la Universidad Autónoma del Estado de Morelos.

\section{Referencias}

1. Miller AB, Chamberlain J, Day NE, Hakama M, Prorok PC. Report on a W orkshop of the UICC Project on Evaluation of Screening for $C$ ancer. Int J Cancer 1990;46:761-769.

2. Hakama M, C hamberlain J, D ay N E, Miller AB, Prorok PC. Evaluation of screening programms for gynaecological cancer. $\mathrm{Br}$ J Cancer 1985;52: 669-673.

3. Benedet JL, Anderson GH, Matisic JP. A comprehensive program for cervical cancer detection and management. Am J 0 bstet Gynecol 1992; 166:1254-1259.

4. Lazcano-Ponce E, Buiatti E, Nájera P,Alonso P, Hernández M. Evaluation model of the Mexican $\mathrm{N}$ ational Program for Early Cervical Cancer Detection and proposals for a new approach. Cancer C auses Control 1998; 9:241-251. 
5. Harlan LC, Bernstein AB, Kessler LG. Cervical cancer screening:W ho is not screened and why? Am J Public Health 1991;81:885-890.

6. Peters RK, Bear MB,Thomas D. Barriers to screening for cancer of the cervix. Prev Med 1989;18:133-146.

7. Morrell D. Perceptions and opinions on the performance of Pap smears: A survey of clinicians using a commercial laboratory.Am J Prev Med 1996; 12(4):271-276.

8.W ard J, D onnelly $N$, Holt P. Impact in general practice of the policies of the organised approach to preventing cancer of the cervix. Aust N Z J Public Health 1998;22 suppl 3:336-341.

9. Cohen MM. W hy is there no progress against cervical cancer? CMAJ 1996;154(12):1867-1869.

10. Stata Corporation. 1993 Stata reference manual. Release 3.1. 6a edición. College Station, TX: Stata Corporation,1993.

11. Burack R, Gimotty P, G eorge J, McBride S, Moncrease A, Simon M, et al. How reminders given to patients and physicians affected Pap smear use in a health maintenance organization. Results of a randomized controlled trial. Cancer 1998:82(12):2391-2400.

12. Martenson D. El aprendizaje en las facultades de medicina: una perspectiva sobre la situación actual. Educ Med 1998;(1)1:7-12.

13. Ragland D, Buffler P, Reingold A, Syme L, Buffler M. D isease and injury in California with projections to the year 2007. West J Med 1998;168: 378-399.

14. IARC. W orking group on evaluation of cervical cancer screening programmes. Screening for squamous cervical cancer: Duration of low risk after negative results of cervical cytology and its implication for screening policies. BMJ 1986;293:659-664.

15. MacG regor JE. Timing of cervical smears. BMJ 1977;1:774-775.

16. Fruchter RG, Boyce J, Hunt M. Missed opportunities of early diagnosis of cancer of the cervix.Am J Public Health 1980;70:418-420.

17. N orman SA, Talbott E, Kuller L, Stolley P, D unn M, Baffone K et al.The relationship of Papanicolaou testing and contacts with the medical care systems to stage at diagnosis of cervical cancer. Arch Intern Med 1991; 151:58-64

18. Lazcano $E, N$ ájera $P, A$ lonso $P, B$ Buatti E, Hernández M. Programa de D etección 0 portuna de Cáncer Cervical en México. I. Diagnóstico situacional. 1996;42(3):123-140.
19. W ard J, Gordon J, Sanson-Fisher R. Strategies to increase preventive care in general practice. Med J Aust 1991;154:523-531.

20. Lewis $D$, Mitchell $H$. An evaluation of cervical screening in general practice. Med J Aust 1994;160:628-632.

21. Hermens RP, Hak E, Hulscher ME, Mulder J, Braspenning JC, Grol RP. $D$ o general practices adhere to organizational guidelines for effective cervical cancer screening? Fam Pract 1998;15(2):112-118.

22. Young JM, W ard JE. Strategies to improve cancer screening in general practice: Are guidelines the answer? Fam Pract 1999;16(1):66-70.

23. Monnet E, Mauny F, Marquant A, Michaud C, Ferry JP. Knowledge and participation of general practitioners in cervical cancer screening: Survey in a French pilot area. Rev Epidemiol Sante Publique 1998:46(2):108-114. 24. Programa Nacional Indicativo de Posgrado. Cuadernos de Modernización Educativa 1989-1993;(6): anexo A. México, D.F.: SEP, 1993.

25. $\mathrm{N}$ arro J. La U niversidad y la educación continua en el área de la salud. En:Reunión Latinoamericana de Educación Continua en el Area de la Salud en el Primer N ivel de Atención. México, D.F.: UN AM, 1986.

26. O rganización Panamericana de la Salud. Educación Continua. Informe de un grupo de consulta sobre los programas de educación continua para el personal de salud en A mérica Latina y el Caribe.W ashington, D.C: 0 rganización Panamericana de la Salud, 1975.

27. A réchiga H. Educación médica continua (Introducción). Gac Med Mex 1996; 132 suppl 2:141-143.

28. Palma V, D elgado H, Fischer M. IN C AP:Actualización de conocimientos médicos, utilizando técnicas de educación a distancia. Educ Med Salud 1991;25:315-325.

29. Sarramona I, López J. Un ejemplo de programa autoformativo dirigido a médicos generalistas en servicio. Rev Iberoamericana Educ Distancia 1998;(1):147-154.

30. N ickerson R. Enseñar a pensar. Barcelona: Paidós, 1994.

31.Vernon DTA, Blake RL. D oes problem-based learning work? A metaanalysis of evaluative research. Acad Med 1993;68(7):550-553.

32.Albanese MA, Mitchell S. Problem-based learning:A review of literature on its outcomes and implementation issues. A cad Med 1993;68: 52-81.

33. Programa de Prevención y Control del Cáncer Cervicouterino 19982000. México, D.F.: SSA, 1998. 